\title{
Livros Infantis para Prevenção do Abuso Sexual Infantil: Uma Revisão de Estudos
}

\author{
Sheila Maria Prado Soma ${ }^{1}$ \\ Lúcia Cavalcanti de Albuquerque Williams \\ Departamento de Psicologia da Universidade Federal de São Carlos, \\ São Carlos, São Paulo, Brasil
}

\begin{abstract}
Resumo
A literatura pode ser uma ferramenta para inspirar, encorajar, informar e potencialmente promover mudanças na vida das crianças. Em diversos países existem livros que abordam o tema do abuso sexual. Tais livros têm potencial para aumentar a conscientização das crianças a respeito dessa temática. Portanto, é fundamental analisar esses LIAPs (Livros Infantis de Abordagem Preventiva) antes de empregá-los em programas preventivos ou ainda antes de oferecê-los como leitura. Dessa forma, o presente trabalho consiste em uma revisão de literatura científica sobre a avaliação de livros infantis que tratam da temática do abuso sexual. Nessa busca, foram encontrados 31 artigos relacionados aos descritores, entretanto apenas três estudos tratavam especificamente sobre a análise de livros infantis. Os estudos analisaram os livros utilizando critérios pré-estabelecidos. De uma forma geral, os estudos apontaram que os livros analisados tinham grande valor para a prevenção e proteção de crianças em relação ao abuso sexual, ainda que nenhum estudo tenha avaliado empiricamente a utilização de tais livros. Foram descritos pontos em comum em cada estudo e identificada a necessidade de produzir estudos semelhantes no Brasil.
\end{abstract}

Palavras-chave: Livros infantis, abuso sexual, prevenção.

\section{Children's Books for Prevention of Child Sexual Abuse: A Review of Studies}

\begin{abstract}
Literature may be a powerful tool to inspire, encourage, inform and potentially promote changes in children's lives. There are numerous books on the subject of sexual abuse in many countries. These books have the potential to increase children's awareness to this subject. Therefore, it is extremely important to analyze these materials before using them in preventive programs or before offering them to children. Thus, the present paper aims to review the scientific literature on assessment of children's books that deal with the topic of sexual abuse, emphasizing the main ones. In this search, we found 31 articles related to the descriptors, however only three studies dealt analyzed specifically children's books. The studies examined the books based in pre-established criteria. In general, the studies showed that the analyzed books had great value for prevention and protection of children against sexual abuse, although no studies were found which empirically assessed interventions using such books. Common points were described in each study, and the need for similar studies in Brazil was identified.
\end{abstract}

Keywords: Children books, sexual abuse, prevention.

Endereço para correspondência: Rua Javari, 4943, casa 12, Geraldo de Carvalho, Ribeirão Preto, SP, Brasil 14061-310. E-mail: sheila.soma@yahoo.com.br e williams@ufscar.br

Apoio: Processo n²013/02668-9, Fundação de Amparo à Pesquisa do Estado de São Paulo (FAPESP). 


\section{Libros Infantis para la Prevencíon del Abuso Sexual Infantiles: Una Revisíon de Estudios}

\section{Resumen}

La linteratura puede ser una herramienta poderosa para inspirar, animar, informar y, potencialmente, promover cambios en la vida de los niños y niñas. En muchos paises hay numerosos libros sobre abuso sexual. Estós libros tienen el potencial de aumentar la conciencia de los niños y ninãs sobre este tema. por lo tanto, es de importancia fundamental analizar estos materiales antes de emplearlos en programas de prevención o mismo antes de ofrecerlos como lectura. Asi, el presente trabajo tiene como objetivo ofrecer una revisión de la literatura científica sobre la evaluacion de libros sobre abuso sexual para ninõs y niñas. En esta búsqueda, fueron encontrados 31 artículos relacionados con los descriptores, pero solo tres estudios trataron especificamente con el análisis de los libros de los niños y ninãs. Los estudios examinaron los libros de un través de criteria preestablecidos. En general los estudios demostraron que los libros analizados tenian gran valor para la prevención y protección de los niños y ninãs contra el abuso sexual, aunque no se soncontraran estúdios empíricos que evaluaram el uso destes libros. Se describieron comunmente en cada estudio, y señaló la necesidad de realizar estudios semejantes estos en Brasil.

Palabras clave: Libros infantiles, abuso sexual, prevencion.

"O livro é aquele brinquedo, por incrível que pareça que, entre um mistério e um segredo põe ideias na cabeça". Maria Dinorah Luz do Prado

O abuso sexual é considerado uma das formas mais graves de violência praticada contra crianças e adolescentes. É um fenômeno universal que atinge todas as idades, níveis sociais, etnias, gêneros, culturas e religiões e suas consequências acarretarem muitos efeitos negativos ao desenvolvimento das crianças e adolescentes vitimizados, necessitando de medidas de enfrentamento em todos os níveis: Familiar, Social, Saúde, Educação, Político e Judicial. Tal fenómeno compreende o envolvimento de uma criança ou adolescente em algum tipo de atividade sexual para a qual não está preparado(a) e que não consegue ou não pode consentir. É praticado por um adulto, criança ou adolescente que, por sua fase de desenvolvimento psicossexual mais adiantado, apresenta-se numa posição na qualhá diferença de poder e força física, de responsabilidade e/ou confiança em relação à sua vítima. A pessoa que ofende sexualmente a outra busca sua satisfação sexual junto a sua vítima utilizando-se da coerção, indução ou força (Ministério da Educação, 2004, 2011; World Health Organization [WHO], 2002).
O abuso sexual infantil compreende o envolvimento de crianças e adolescentes em situações nas quais há contato sexual físico (sexo oral, carícias e toques genitais) podendo chegar à interação sexual completa (sexo genital ou anal), como também pode ocorrer sem o contato físico (assédio sexual, abuso sexual verbal, telefonemas obscenos, produção e exposição a pornografia, bem como voyerismo e exibicionismo), além da exploração sexual com finalidade de lucro (Ministério da Educação, 2004, 2011; Ministério da Saúde, 2002; WHO, 2002).

$\mathrm{O}$ (a) ofensor(a) sexual geralmente tem preferência por vítimas com autoestima baixa, inseguras e que necessitam ser cuidadas, o que as torna suscetíveis a condições de sujeição e condicionamento amplamente exploradas pelos(as) ofensores(as). Estando vulneráveis, as vítimas apresentam dificuldades em discriminar uma possível situação abusiva, bem como um(a) potencial ofensor(a), daí a importância dos programas preventivos e da utilização de livros que abordam a temática do abuso sexual infantil (ASI) que possam ensinar habilidades protetivas para crianças reconhecerem e se protegerem diante de situações abusivas (Padilha \& Gomide, 2004; Padilha \& Williams, 2009).

Diversas estratégias podem ser utilizadas para prevenir o abuso sexual infantil, dentre elas 
a utilização de livros ilustrados. Um livro de história leva a criança a interagir com o enredo e as personagens, dialogar com as mesmas e identificar-se. Ao contar histórias é possível incentivar a criatividade das crianças, possibilitar que elas vivenciem o ambiente das personagens, se identifiquem com elas e conheçam seu mundo. Para ouvir histórias não é necessário estar alfabetizado, pois a criança desde cedo tem contato com textos de forma oral, pela voz dos pais e avós quando lhes são ditos contos de fadas, histórias inventadas, textos bíblicos, poemas dentre outros (Abramovich, 1993).

As histórias podem ter duas funções principais: a literária que tem a função de provocar emoções e encantamento e a pedagógica, que serve como instrumento para promover a aquisição de novas habilidades. O desafio então, é que as histórias possam apresentar às crianças a realidade que as cerca sem perder a ludicidade $\mathrm{e}$ se afastar da arte (Caldin, 2002).

A literatura pode ser uma ferramenta para inspirar, encorajar, informar e potencialmente promover mudanças na vida das pessoas (McDaniel, 2001). Ela pode auxiliar as crianças a se tornarem mais conscientes da realidade que as cerca, principalmente quando se trata de crianças que vivem situações difíceis. $\mathrm{O}$ enredo de uma história pode proporcionar aprendizado para a criança pelos conflitos dos personagens, suas angústias e alegrias, auxiliando-a a encontrar soluções para suas dificuldades e desafios ou apenas para diverti-las (Craveiro, 2009).

As histórias também transmitem cultura, reproduzindo padrões de comportamento ou regras vigentes em determinada comunidade verbal e indicando um caminho a seguir. Essas regras presentes nas histórias estão diretamente relacionadas aos fatos vivenciados pelos personagens e têm a finalidade de ajudar crianças a aprenderem novos comportamentos, podendo influenciar os jovens leitores (McDaniel, 2006; Vasconcelos et al., 2006).

Os livros com conteúdos preventivos permitem que as crianças explorem e partilhem experiências, auxiliando-as a ampliar seu entendimento sobre as complexidades da vida e do mundo e impelindo-as a enxergar a realidade sob novas perspectivas e circunstâncias. Ao apresentar conteúdos que incentivem a reflexão e a aprendizagem sobre os problemas da vida cotidiana, as histórias trazem informações importantes para que as crianças possam reconhecer cenários potencialmente prejudiciais ou ainda identificar sua própria vitimização e, principalmente, a procurar ajuda. Por meio da leitura, a criança tem oportunidade de perceber que não é o único ser no mundo que passa por dificuldades, ou seja, descobre que há outras crianças que enfrentam situações semelhantes (McDaniel, 2001).

Os livros infantis apresentam várias classificações, dentre elas estão aqueles cujos enredos refletem normas vigentes, papeis sociais e de gênero, abordando temas como violência, abuso de substancias, dentre outros. Esses livros, geralmente são classificados como pedagógicos, pois são utilizados como instrumento útil ao aprendizado de comportamentos relevantes, entretanto, essa classificação não contempla todo o potencial desses livros. Dessa forma, a sigla LIAP (Livros Infantis de Abordagem Preventiva) foi cunhada pelas presentes autoras para classificar livros que proporcionam às crianças representações da vida cotidiana, sendo especialmente úteis para o ensino sobre situações específicas e abordagem de temas embaraçosos e difíceis. É importante lembrar, que esse tipo de livro ainda não é amplamente divulgado, pois geralmente os pais e responsáveis têm receio de incentivar tal leitura às crianças, por medo de que percam a inocência, ou ainda que se sintam incentivadas e curiosas a respeito de determinados temas relacionados a sexo, desenvolvimento sexual e abuso de drogas (Hollander, 1992; McDaniel, 2001; Tomlinson, 1995). Contudo, uma das dificuldades apresentadas por crianças vitimizadas sexualmente é o fato de que não conseguem discriminar as manifestações afetivas; elas não percebem a diferença entre o que é um ato agressivo e um ato cooperativo não sabendo como agir de forma adequada frente a estas situações. Portanto, aprender a identificar riscos deve ser um dos objetivos principais dos LIAPs (Caminha, 2002). 
Em diversos países existem livros para crianças e jovens que abordam o tema do abuso sexual. Tais livros tem o potencial para aumentar a conscientização das crianças a respeito dessa temática. De uma forma geral, esses LIAPs, bem como os programas de prevenção, devem minimamente informar as crianças sobre o que é o abuso sexual e que elas podem dizer não às investidas do(a) ofensor(a), devendo informar ainda a quem recorrer caso uma situação semelhante ocorra. Adicionalmente, tais livros precisam oferecer informações a respeito da posse do próprio corpo, dos tipos de toque e não perpetuar estereótipos tanto a respeito da vítima quanto do(a) ofensor(a) (Hollander, 1992; Williams, Padilha, \& Brino, 2013; Wolfe, 2006; Wurtele, 2008).

Contudo, há uma carência de informações sobre a adequação desses LIAPs e sobre sua eficácia a respeito dos objetivos a que se propõem que são: informar, prevenir e identificar casos de abuso sexual infantil. Portanto é de fundamental importância analisar os LIAPs destinados às crianças antes de empregá-los em programas preventivos ou ainda antes de oferecê-los como leitura (Hollander, 1992). Dessa forma o presente trabalho pretende realizar uma revisão de literatura científica sobre avaliação de livros infantis que tratam da temática do abuso sexual.

\section{Método}

O objetivo desta revisão foi o de verificar a ocorrência de estudos que avaliem livros infantis para prevenção do abuso sexual em bases eletrônicas de dados (INDEXPsi, Web of Cience, Pepsic, SciELO, PUBMed, PsycNET). Foram consultados de forma restrospectiva todos os estudos realizados entre os anos de 1997 e 2012 utilizando as palavras-chave: "livros de imagens" (picture books, libros de imagens), "livros infantis" (children books, libros para crianças) e "abuso sexual infantil" (child sexual abuse, abuso sexual de crianças), a busca limitou-se aos estudos publicados em inglês, espanhol e português.

Os artigos foram identificados pelos títulos, palavras chave e leitura dos resumos (abstracts e resumen), tendo como critérios de inclusão: (a) estudos que avaliassem livros infantis sobre abuso sexual e (b) estudos que utilizassem livros infantis em programas de prevenção do abuso sexual infantil.

Após a seleção dos textos, os resultados foram organizados em uma tabela contendo as seguintes informações: (a) título; (b) quantidade de vezes em que o artigo apareceu nas buscas; (c) palavras-chave; (d) resumo (abstract e resumen); (e) periódico hospedado; (f) tema e principais informações contidas no texto.

Foram excluídos os artigos que apresentavam narrativas de adolescentes sobre sua vitimização; biografia de pessoas vítimas; comentários de livros sobre abuso sexual; comentários sobre mulheres vítimas; relatos de histórias autobiográficas sobre abuso sexual; alienação parental; intervenção terapêutica em casos de abuso sexual; relação entre abuso sexual infantil e abuso sexual na idade adulta; perversão na infância; maus-tratos infantil; violência íntima entre parceiros e violência familiar; artigos escritos em idioma diferente do inglês, português ou espanhol e artigos com informações repetidas e presentes em outros artigos. Quando os títulos e resumos não eram esclarecedores, o texto era lido na íntegra com o objetivo de melhor compreender o estudo.

Foram selecionados 31 artigos que estavam relacionados aos descritores (palavras-chave). Deste total, 28 artigos foram excluídos porque não satisfizeram os critérios de inclusão, restando apenas três.

\section{Resultados e Discussão}

Os resultados obtidos na revisão sistemática estão representados na Tabela 1 com o objetivo de descrever de forma breve o conteúdo dos estudos de Lampert (2011), Lampert e Walsh (2010) e McDaniel (2001). 
Tabela 1

Comparação entre os Estudos Analisados

\begin{tabular}{|c|c|c|c|c|c|c|}
\hline Estudo & Objetivo & $\begin{array}{l}\text { Seleção do } \\
\text { material }\end{array}$ & $\begin{array}{l}\text { Critérios de } \\
\text { análise }\end{array}$ & Resultados & Principais problemas & $\begin{array}{c}\text { Principais } \\
\text { contribuições }\end{array}$ \\
\hline $\begin{array}{l}\text { McDaniel } \\
(2001)\end{array}$ & $\begin{array}{c}\text { Ajudar adultos } \\
\text { averiguar } \\
\text { os LIAPs } \\
\text { mais } \\
\text { benéficos } \\
\text { para a } \\
\text { prevenção } \\
\text { de ASI }\end{array}$ & $\begin{array}{c}\text { Livros obtidos } \\
\text { em bibliotecas } \\
\text { e livrarias para } \\
\text { crianças de } \\
\text { 4-8 anos }\end{array}$ & $\begin{array}{c}9 \text { critérios } \\
\text { estabelecidos } \\
\text { por Rudman } \\
\text { (1985) }\end{array}$ & $\begin{array}{c}\text { Descreve } \\
\text { e analisa } \\
13 \text { livros } \\
\text { encontrados } \\
\text { e tece } \\
\text { comentários. } \\
\text { Aponta } 3 \\
\text { livros como } \\
\text { sendo mais } \\
\text { úteis para } \\
\text { ensinar } \\
\text { crianças } \\
\text { a respeito } \\
\text { do que } \\
\text { é ASI }\end{array}$ & $\begin{array}{c}\text { Não detalha o método } \\
\text { utilizado para } \\
\text { selecionar os livros }\end{array}$ & $\begin{array}{c}\text { Acrescenta } \\
4 \text { critérios } \\
\text { adicionais } \\
\text { considerados } \\
\text { importantes } \\
\text { para futuras } \\
\text { análises }\end{array}$ \\
\hline $\begin{array}{l}\text { Lampert, } \\
\& \text { Walsh } \\
(2010)\end{array}$ & $\begin{array}{c}\text { Servir de base } \\
\text { para o desen- } \\
\text { volvimento } \\
\text { de um corpus } \\
\text { de literatura } \\
\text { infantil para } \\
\text { lidar com } \\
\text { o ASI e } \\
\text { proporcionar } \\
\text { um } \\
\text { levantamento } \\
\text { desse tipo } \\
\text { LIAPs para } \\
\text { explorar seu } \\
\text { conteúdo em } \\
\text { diferentes } \\
\text { contextos }\end{array}$ & $\begin{array}{c}\text { Busca em } \\
\text { banco } \\
\text { de dados } \\
\text { eletrônico } \\
\text { por catálogos } \\
\text { de livros de } \\
\text { bibliotecas } \\
\text { com a } \\
\text { utilização de } \\
\text { palavras-chave } \\
\text { em inglês, } \\
\text { para crianças } \\
\text { de 3-8 anos, } \\
\text { livros com } \\
\text { menos de } \\
50 \text { páginas } \\
\text { e ilustrações } \\
\text { coloridas }\end{array}$ & $\begin{array}{l}\text { Dois critérios } \\
\text { de análise: } \\
\text { (a) } 18 \text { dos } 32 \\
\text { critérios das } \\
\text { Diretrizes para } \\
\text { Programas de } \\
\text { Redução de } \\
\text { Vitimização } \\
\text { da Criança } \\
\text { (Hawkins, } \\
\text { 1999); (b) } \\
\text { Análise } \\
\text { literária }\end{array}$ & $\begin{array}{l}\text { A busca } \\
\text { resultou em } 58 \\
\text { exemplares. } \\
\text { Por meio de } \\
\text { uma seleção } \\
\text { aleatória } \\
\text { chegou-se } \\
\text { ao total de } 15\end{array}$ & $\begin{array}{l}\text { Seleção aleatória pode } \\
\text { não ter contemplado } \\
\text { exemplares importantes. } \\
\text { Ao mencionar os } 32 \\
\text { critérios de Hawkins } \\
\text { (1999), os autores } \\
\text { não especificam quais } \\
\text { critérios são esses. } \\
\text { Não fica claro como } \\
\text { os autores chegaram } \\
\text { aos } 18 \text { critérios } \\
\text { selecionados. } \\
\text { Resultados contêm } \\
\text { expressões vagas } \\
\text { na medida em } \\
\text { que não identifica } \\
\text { numericamente (na } \\
\text { maioria, poucos, etc). } \\
\text { Não apresenta quais } \\
\text { os livros são indicados } \\
\text { para programas } \\
\text { preventivos. }\end{array}$ & $\begin{array}{c}\text { Utilização } \\
\text { de critérios } \\
\text { de avaliação } \\
\text { permite uma } \\
\text { visão geral } \\
\text { dos LIAPs }\end{array}$ \\
\hline $\begin{array}{l}\text { Lampert } \\
(2011)\end{array}$ & $\begin{array}{l}\text { Examinar } \\
\text { como os } \\
\text { ofensores } \\
\text { sexuais são } \\
\text { apresentados } \\
\text { e ilustrados } \\
\text { nos livros, } \\
\text { suas } \\
\text { ausências e } \\
\text { invisibilidades }\end{array}$ & $\begin{array}{l}\text { "60 e tantos" } \\
\text { livros sobre } \\
\text { ASI obtidos } \\
\text { por doação }\end{array}$ & $\begin{array}{l}5 \text { critérios } \\
\text { estabelecidos } \\
\text { pela autora }\end{array}$ & $\begin{array}{c}\text { Foram } \\
\text { selecionados } \\
8 \text { livros por } \\
\text { se tratarem } \\
\text { de livros } \\
\text { ilustrados para } \\
\text { crianças que } \\
\text { continham } \\
\text { uma descrição } \\
\text { escrita ou por } \\
\text { ilustração dos } \\
\text { perpetradores } \\
\text { do abuso. }\end{array}$ & $\begin{array}{c}\text { A descrição da forma } \\
\text { como os livros foram } \\
\text { selecionados é vaga } \\
\text { sem a descrição clara } \\
\text { do tamanho da amostra. } \\
\text { Não apresenta quais } \\
\text { os livros mais } \\
\text { indicados para } \\
\text { programas } \\
\text { preventivos. }\end{array}$ & $\begin{array}{c}\text { Utilização } \\
\text { de critérios } \\
\text { de avaliação } \\
\text { permite uma } \\
\text { visão geral } \\
\text { dos LIAPs }\end{array}$ \\
\hline
\end{tabular}


Em relação aos objetivos, os estudos de Lampert e Walsh (2010) e McDaniel (2001) apresentam semelhanças, pois de uma forma geral, ambos pretendem identificar os LIAPs com maior potencial para prevenção do abuso sexual. Em contraste, o estudo de Lampert (2011) pretende analisar a forma pela qual os(as) ofensores(as) sexuais são representados nas obras infantis.

Os estudos de Lampert (2011), Lampert e Walsh (2010) e McDaniel (2001) apresentam em sua proposta a intenção de fornecer subsídios para selecionar os melhores LIAPs para serem utilizados em programas preventivos, entretanto, os estudos de Lampert (2011), Lampert e Walsh (2010) e McDaniel (2001) não apresentam uma análise quantitativa que possa indicar de forma clara os LIAPs que obtiveram um melhor desempenho para tal finalidade. Contudo o estudo de McDaniel (2001) indica com base em análise qualitativa que três, dos 13 livros analisados têm um maior potencial para prevenção.

Em relação ao método de seleção dos livros a serem analisados, os estudos de Lampert e Walsh (2010) e McDaniel (2001) realizaram buscas em bases de dados online, bem como em bibliotecas. O estudo de Lampert (2011) realizou suas buscas em livros doados. Entretanto, os estudos não citam de forma clara e específica quais bases foram utilizadas e qual o período em que as buscas foram realizadas. Apenas o estudo realizado por Lampert e Walsh (2010) apresentou busca por palavras chave e um processo de seleção dos livros mais preciso (busca em banco de dados eletrônico por catálogos de livros de bibliotecas; com a utilização de palavras chave em inglês; para crianças de 3 a 8 anos; livros com menos de 50 páginas e ilustrações coloridas)..

A falta de critérios em relação ao método de seleção dos livros compromete a qualidade dos estudos, principalmente porque uma escolha aleatória ou com critérios imprecisos impossibilita a replicabilidade do estudo, não sendo possível avaliar sua evidência científica.

Outro fator que compromete a qualidade dos estudos de Lampert (2011), Lampert e Walsh (2010) e McDaniel (2001), é o fato de a análise dos livros ter sido realizada pelos próprios autores, o que não permite que seja realizado um teste de confiabilidade dos resultados obtidos, como acontece quando juízes especialistas são convidados a realizar suas análises.

Em se tratando da forma como os livros foram analisados, os estudos de Lampert (2011), Lampert e Walsh (2010) e McDaniel (2001) refletem a mesma metodologia de análise pelo estabelecimento de critérios específicos, o que é um procedimento muito útil nesses casos.

No estudo de McDaniel (2001) são utilizados 9 critérios estabelecidos por Rudman (1985) (como citado por McDaniel, 2001) que são: (a) nunca culpar a vítima; (b) ser cuidadoso e indicar o ofensor como um conhecido da vítima ao invés de um estranho; (c) refletir sobre o fato de que os ofensores não tem um estereótipo, podendo ser de qualquer etnia, classe social ou gênero; (d) procurar diferenciar estupro de outros abusos sexuais, diferenciando ainda carícias abusivas de toques afetuosos; (e) evitar soluções simples, que não são úteis ou realistas, pois não existem finais felizes sem esforço; (f) incluir informações para ajudar os leitores a reconhecer se os mesmos são vítimas de abuso; (g) ensinar crianças a diferenciarem entre os segredos que são potencialmente ameaçadores para a sua segurança e surpresas para agradar alguém; (h) sugerir formas alternativas de obtenção de ajuda; e (i) evitar cenas gráficas de abuso e violência.

McDaniel (2001), acrescenta ainda, quatro critérios para contribuir com a análise dos LIAPs que são: (a) apresentar modelos positivos; (b) desmistificar o sexo e contribuir para atitudes saudáveis em relação à sua sexualidade e seu corpo; (c) ensinar o pensamento crítico e habilidades de tomada de decisão; e (d) apresentar personagens com que os leitores possam se identificar.

Lampert e Walsh (2010), utilizaram 18 dos 32 critérios estabelecidos por Hawkins (1999) que constituem diretrizes para programas de redução da vitimização da criança. São eles: (a) Ensinar às crianças normas de segurança geral (endereço, telefone, telefones de emergência, etc); (b) fornecer instruções explícitas sobre a posse de seu próprio corpo (meu corpo me pertence); (c) ajudar às crianças a distinção entre um toque adequado e inadequado; (d) ajudar às 
crianças a distinguir a diferença entre segredos e surpresas, ressaltando que alguns segredos devem ser informados; (e) ensinar à criança que ela pode dizer não e rejeitar insinuações não desejadas; (f) incentivar a criança a relatar a um adulto de confiança sobre coisas que a incomodam; (g) ensinar a criança a identificar pessoas de sua confiança; (h) ensinar sobre as partes íntimas e anatomia de seu corpo; (i) fornecer a exposição repetida de mensagens de segurança; $(j)$ ensinar que os adultos às vezes agem de forma inadequada ou inapropriada; (k) salientar que o comportamento inadequado de um adulto nunca é culpa da criança; (1) ensinar que as vítimas não têm um estereótipo, ou seja, podem ser de qualquer gênero, idade e etnia; (m) ensinar que não existe um estereótipo de agressor, que o mesmo pode ser um adulto do sexo masculino ou feminino, ou ainda uma criança maior ou adolescente; (n) ensinar que os agressores podem ser pessoas de sua confiança ou pessoas conhecidas; (o) incentivar a denúncia e a notificação dos casos; (p) salientar que as crianças têm o direito de ficarem em segurança; (q) oferecer oportunidade para a participação ativa das crianças durante a leitura; e (r) fornecer material de apoio para pais e professores.

Refinando suas análises, utilizaram ainda análise literária dos livros que correspondeu em verificar as estratégias linguísticas dos LIAPs, como a escolha das palavras, focalização e ponto de vista, estrutura narrativa e o simbolismo que eram aplicados a cada um dos livros, além do tratamento de suas ilustrações.

Ao considerar a forma como os ofensores do abuso sexual são representados nas obras ilustradas Lampert (2011) utilizou 5 critérios para nortear sua análise: (a) representações dos(as) autores(as); (b) tabus: raça, classe social; (c) tabus: efeitos físicos de abuso infantil; (d) descrições dos(as) ofensores(as): traços, relacionamentos, motivações e (e) fechamentos e problemáticas.

Os resultados nos estudos de Lampert (2011), Lampert e Walsh (2010) e McDaniel (2001) são apresentados de forma qualitativa, com descrições de cada um dos livros analisados de acordo com os critérios especificados em cada um dos estudos. De uma forma geral, como resultado, os três estudos buscaram descrever quais critérios eram contemplados ou não por cada um dos LIAPs analisados, numa tentativa de identificar quais deles tinham maior potencial para serem utilizados para prevenção do abuso sexual infantil. Contudo, uma análise quantitativa forneceria dados importantes a respeito da porcentagem de critérios atingida por cada um dos LIAPs, fornecendo uma análise comparativa entre as obras, o que traria maior fidedignidade e clareza aos resultados.

Os estudos de Lampert (2011), Lampert e Walsh (2010) e McDaniel (2001) trazem contribuições relevantes, pois são referências para pesquisas posteriores que pretendam avaliar o conteúdo desses LIAPs para garantir a aquisição de habilidades protetivas nas crianças que venham utilizá-los. Podem contribuir ainda, para que autores elaborem e/ou revisem suas obras com a finalidade de potencializar o efeito das mesmas para auxiliar pais e educadores na seleção de LIAPs para as crianças ou programas de prevenção. De forma geral, os estudos apontaram que os livros analisados tinham grande valor para a prevenção e proteção de crianças em relação ao abuso sexual.

\section{Considerações Finais}

Por se tratar de um material direcionado para crianças e por abordar um tema difícil, é preciso ter cuidado ao oferecer esse tipo de leitura e, portanto, as análises dos LIAPs são relevantes.

É importante destacar ainda que os três estudos foram realizados em países como Austrália (Lampert, 2011; Lampert \& Walsh, 2010) e Estados Unidos (McDaniel, 2001), o que significa que no Brasil não foram encontradas pesquisas que tratem do tema. Em contrapartida, há uma gama de LIAPs disponíveis em nossas livrarias e bibliotecas, obras traduzidas e também LIAPs produzidos por autores brasileiros como, por exemplo: Pipo e Fifi (Arcari, 2013), Antônio (Ferreira, 2012), O Segredo da Tartanina (Silva, Soma, \& Watarai, 2011), Segredo Segredíssimo (Barros, 2011) e Chapeuzinho Cor-de-rosa e a Astúcia do Lobo Mau (Siquinel, 2010). Tais li- 
vros carecem de análise de conteúdo semelhantes às propostas nos estudos apresentados nesse artigo, indicando uma lacuna a ser preenchida considerando a relevância do tema.

Para preencher essa lacuna, dois estudos nessa área estão sendo realizados pelas presentes autoras. O primeiro tem o objetivo de verificar se a contação de uma história infantil específica sobre abuso sexual, tem potencial para promover a aquisição de habilidades de proteção em crianças de 7 a 10 anos de idade em comparação com outro livro não específico e um grupo controle. O segundo estudo pretende avaliar os LIAPs publicados por autores brasileiros entre os anos de 2010 e 2013, de acordo com critérios como os propostos por Lampert (2011), Lampert e Walsh (2010) e McDaniel (2001) em seus estudos.

Os estudos aqui descritos podem servir de base para outros como, por exemplo, estabelecer critérios de avaliação dos LIAPs adaptados para a população brasileira, ou ainda analisar aspectos específicos dos livros, como a maneira pela qual a situação de abuso sexual é representada, quais as consequências do abuso sexual descritas, como as questões legais são tratadas nesses LIAPs e como as vitimas são representadas, dentre tantos outros aspectos.

\section{Referências}

Abramovich, F. (1993). Literatura infantil: Gostosuras e bobices. São Paulo, SP: Scipione.

Arcari, C. (2013). Pipo e Fifi: Prevenção de violência sexual na infância. Recuperado em 20 de setembro, 2013, de http://www.pipoefifi.org.br/home. html

Barros, O. (2011). Segredo segredíssimo. São Paulo, SP: Geração Editorial.

Caldin, C. F. (2002). A oralidade e a escritura na literatura infantil: Referencial teórico para a hora do conto. Revista Eletrônica de Biblioteconomia e Ciência da Informação, 7(13), 25-38.

Caminha, R. M. (2002). Grupoterapia cognitivo-comportamental em abuso sexual infantil. In $\mathrm{H}$. J. Guilhardi, B. B. P. Madi, P. P. Queiroz, \& M. C. Scoz (Eds.), Sobre comportamento e cognição: Contribuições para a construção da teoria do comportamento (pp. 322-335). Santo André,
SP: Esetec.

Craveiro, C. C. (2009). Efeito da exposição continuada a regras descritivas sobre o comportamento escolar de crianças (Dissertação de mestrado, Universidade Federal do Pará, Belém, PA, Brasil).

Ferreira, H. M. (2012). Antônio. Rio de Janeiro, RJ: Escrita Fina.

Hawkins, M. (1999). Guidelines for programs to reduce child victimization: A resource for communities when choosing a program to teach personal safety to children. Retrieved September 20, 2013, from http://files.eric.ed.gov/fulltext/ ED465464.pdf

Hollander, S. K. (1992). Making young children aware of sexual abuse. Education Digest, 4(26), 305-318.

Lampert, J. (2011). Sh-h-h-h: Representations of perpetrators of sexual child abuse in picture books. Sex Education: Sexuality, Society and Learning, 12(2), 177-185.

Lampert, J., \& Walsh, K. (2010). 'Keep telling them until someone listens': Understanding prevention concepts in children's picture books dealing with child sexual abuse. Children's Literarure in Education, 41(2), 146-167.

McDaniel, C. (2001). Children's literature as prevention of child sexual abuse. Children's Literarure in Education, 32(3), 203-224.

McDaniel, C. (2006). Critical literacy: A way of thinking, a way of life. New York: Peter Lang.

Ministério da Educação. (2004). Guia escolar: Métodos para identificação de abuso e exploração sexual de crianças e adolescentes. Brasília, DF: Autor.

Ministério da Educação. (2011). Guia escolar: Identificação de sinais de abuso e exploração sexual de crianças e adolescentes. Brasília, DF: Autor.

Ministério da Saúde. (2002). Notificação de maus-tratos contra crianças e adolescentes pelos profissionais de saúde: Um passo a mais na cidadania em saúde. Brasília, DF: Secretaria de Assistência à Saúde.

Padilha, M. G., \& Gomide, P. I. C. (2004). Descrição de um processo terapêutico em grupo para adolescentes vítimas de abuso sexual. Estudos de Psicologia (Natal), 9(1), 53-61.

Padilha, M. G., \& Williams, L. C. (2009). Interven- 
ção escolar para prevenção do abuso sexual com estudantes pré-adolescentes e adolescentes. In L. C. Williams \& E. A. Araújo, Prevenção ao abuso sexual infantil: Um enfoque interdisciplinar (pp. 128-135). Curitiba, PR: Juruá.

Silva, A. R. S., Soma, S. M. P., \& Watarai, C. F. (2011). O segredo da Tartanina: Um livro a serviço da proteção e prevenção contra o abuso sexual infanto-juvenil. Pompéia, SP: Universidade da Família.

Siquinel, C. B. (2010). Chapeuzinho cor-de-rosa e a astúcia do lobo mau. Rio de Janeiro, RJ: LITTRIS.

Tomlinson, C. (1995). Justifying violence in children's literature. In S. Lehr, Batting dragons: Issues and controversy in childrens literature (pp. 39-50). Portsmouth, UK: Heinemann.

Vasconcelos, L. A., Silva, C. C., Curado, E. M., Galvão, P., Naves, A. R., \& Arruda, M. C. (2006). Brincando com histórias infantis: Uma análise do comportamento para o desenvolvimento de crianças. Santo André, SP: Esetec.

Williams, L. C. A., Padilha, M. G. S., \& Brino, R. F. (2013). Programas de prevenção de abuso sexual. In A. P. Serafim, D. M. Barros, \& F. Saffi (Eds.), Temas em Psiquiatria Forense e Psicologia Jurídica III (pp. 231-248). São Paulo, SP:
Vetor.

Wolfe, V. V. (2006). Child sexual abuse. In E. J. Mash \& R. A. Barkley (Eds.), Treatment of childhood disorders (pp. 545-597). New York: The Guilford Press.

World Health Organization. (2002). Global consulation on violence and health violence: A public health priority. Geneva, Switzerland: Author.

Wurtele, S. (2008). Behavioral approaches to educating young children and their parents about sexual abuse prevention. The Journal of Behavior Analysis of Offender and Victim Treatment and Prevention, 1(1) 52-64.
Recebido: 03/10/2013

$1^{a}$ revisão: $16 / 12 / 2013$ Aceite final: 19/12/2013 